\title{
Polymerase Chain Reaction in the Diagnosis and Prognosis of Mediterranean Visceral Leishmaniasis in Immunocompetent Children
}

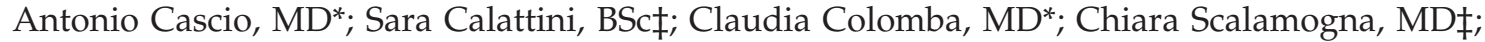

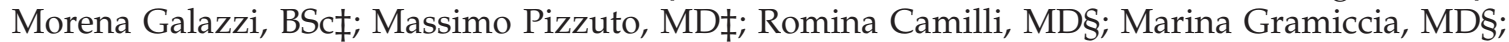 \\ Lucina Titone, $\mathrm{MD}^{*}$; Mario Corbellino, MD ; and Spinello Antinori, MD*
}

\begin{abstract}
Objective. To assess the usefulness of a polymerase chain reaction (PCR) assay amplifying the small subunit rRNA coding region of Leishmania species performed on peripheral blood (PB) and bone marrow (BM) aspirates for the diagnosis and follow-up of visceral leishmaniasis (VL) in children living in the Mediterranean basin.
\end{abstract}

Design. A prospective study was conducted on children consecutively hospitalized over a 1-year period at our Infectious Diseases Department in Sicily (Italy) presenting with fever, hepatosplenomegaly, and/or pancytopenia and a positive Leishmania serology ( $\geq 1: 40)$.

Results. Among the 14 patients hospitalized with signs and symptoms suggestive of the disease and a positive serology, we identified 10 cases of Mediterranean VL. PCR performed on PB and BM aspirates was positive in all cases and concordant with microscopy and/or culture performed on BM. Leishmania DNA was cleared from PB a median of 6 days after the start of treatment; during follow-up (median: 9 months; range: 6-12 months) 1 child relapsed. In this case, BM PCR remained positive with rapid reappearance of a positive signal also in $\mathrm{PB}$.

Conclusions. PB PCR allows a rapid and noninvasive parasitologic diagnosis of Mediterranean VL among immunocompetent children and is at least as sensitive as a diagnosis made on the basis of BM aspirates. The lack of disappearance from $B M$ and the reappearance of positive PCR on PB is predictive of clinical relapse. Qualitative and semiquantitative PCR may be the standard method for monitoring response to therapy in immunocompetent children. Pediatrics 2002;109(2). URL: http://www. pediatrics.org/cgi/content/full/109/2/e27; visceral leishmaniasis, polymerase chain reaction, diagnosis, peripheral blood, bone marrow.

ABBREVIATIONS. VL, visceral leishmaniasis; BMA, bone marrow aspirate; $\mathrm{PB}$, peripheral blood; $\mathrm{PCR}$, polymerase chain reaction; HIV, human immunodeficiency virus; RFLP, restriction fragment length polymorphism; BM, bone marrow.

From the *Istituto di Patologia Infettiva e Virologia, Ospedale dei Bambini G. di Cristina, Università di Palermo, Italy; łIstituto di Malattie Infettive e Tropicali, Ospedale L. Sacco, Università di Milano; and §Istituto Superiore di Sanità, Roma, Italy.

Received for publication Jul 26, 2001; accepted Oct 19, 2001.

Reprint requests to (S.A.) Istituto di Patologia Infettiva e Virologia, Ospedale dei Bambini "G di Cristina", Piazza Montalto 8, Palermo Italy. E-mail: spantin@tin.it

PEDIATRICS (ISSN 0031 4005). Copyright $\odot 2002$ by the American Academy of Pediatrics.
$\mathrm{V}$ isceral leishmaniasis (VL) attributable to Leishmania infantum is a vector-borne zoonotic disease transmitted by sand fly bites and is endemic in rural or periurban areas of the Mediterranean basin. ${ }^{1}$ Before the age-related changes introduced by the acquired immunodeficiency syndrome epidemic in southern European countries, VL mainly affected children younger than 5 years. ${ }^{2,3}$ However, an epidemiologic survey conducted in Sicily between 1987 and 1995 revealed that $53 \%$ of all cases were still observed in children younger than 14 years of age. ${ }^{4}$ In 2 recent retrospective pediatric studies conducted in France and Greece, the median age of patients was 2 years, 5 months.5,6 The most common methods of diagnosing VL are classic parasitologic methods such as microscopic direct examination and in vitro culture of bone marrow aspirates (BMAs). As these techniques require invasive procedures and are difficult to repeat for follow-up of patients, an easy, rapid, and noninvasive method would be valuable. In this regard, polymerase chain reaction (PCR) used on peripheral blood (PB) of patients with VL (mainly human immunodeficiency virus [HIV]-Leishmania coinfected adults) has proved highly specific and sensitive. ${ }^{7-9}$ Therefore, we conducted a prospective study among all children hospitalized for suspected Mediterranean VL during a 1-year period to assess the usefulness of Leishmania PCR in the first diagnosis and follow-up of VL in childhood.

\section{METHODS}

\section{Study Design}

All consecutive patients admitted to the Institute of Infectious Pathology and Virology of the University of Palermo between November 1999 and November 2000 with fever, hepatosplenomegaly pancytopenia, and a positive serology for Leishmania were enrolled in the study. A definitive diagnosis of VL was based on the presence of at least 1 of the following criteria: 1) direct microscopic demonstration of Leishmania amastigotes in the BMA and/or 2) culture isolation of promastigotes from BMAs inoculated into selective medium.

BMAs were performed at the time of diagnosis and 4 weeks after treatment initiation (in the case of confirmed VL); PB samples were collected at the time of diagnosis (T0), every 3 days (T1, T2, T3, T4) for 2 weeks and then at weekly intervals for 2 weeks; thereafter, PB samples were obtained at month 3, 6, 9, and 12 unless a clinical relapse occurred before this period.

In all cases, the DNA extracted from ethylenediaminetetraacetic acid-anticoagulated PB and BMA was assayed for Leishmania species DNA by means of a PCR (that proved highly sensitive and specific) as described elsewhere. ${ }^{9}$ Identification of the Leishmania at the species level was obtained by restriction fragment length 
polymorphism (RFLP) analysis of a repetitive Leishmania-specific nuclear genomic sequence obtained as described by Minodier et al. ${ }^{9-11}$ When available, identification results were compared with the standard isoenzyme characterization data. ${ }^{12}$

Serology was obtained by means of indirect immunofluorescence antibody test using a commercial kit (Leishmania Spot IF, Biomerieux, Marcy L'Etoile, France) and was performed at the time of diagnosis, 4 weeks later, and at each patient's last follow-up visit.

All of the patients with VL were randomly assigned to a specific anti-Leishmania treatment as a part of a multicenter treatment study coordinated by the Istituto Superiore di Sanità, Rome, Italy, and designed to compare a pentavalent antimonial (meglumine antimoniate, Glucantim, Aventis Pharma, Milan, Italy) at a dose of $20 \mathrm{mg} \mathrm{Sb}^{5} / \mathrm{kg}$ /day given intramuscularly for 28 days versus liposomal amphotericin B (Ambisome, Gilead, San Diego, CA) at a dose of $3 \mathrm{mg} / \mathrm{kg} /$ day intravenously for 5 days followed by 1 additional administration after 1 week.

The study was approved by the local ethics committee, and the parents gave informed consent before the enrollment of the children in the study.

\section{RESULTS}

One hundred fourteen PB and 27 bone marrow (BM) samples taken from 14 immunocompetent children were tested. Overall, 10 patients were diagnosed as having VL; the remaining 4 patients (all of whom underwent bone marrow aspiration) had acute leukemia (2 cases) and infectious mononucleosis (2 cases). PCR performed either on PB and BMA in these 4 patients had negative results. Table 1 summarizes the parasitologic, serologic Leishmania zymodeme, and PCR studies conducted on the patients with confirmed VL. In all cases, PCR detected Leishmania in all BM (9/9) and PB (10/10) samples at first diagnosis; semiquantitative PCR analysis showed that the parasite load was at least $1 \log$ higher in the BM samples of 8 cases (Fig 1), and comparable with the $\mathrm{PB}$ and $\mathrm{BM}$ samples of the remaining 2 patients. Microscopy revealed Leishmania amastigotes in 9 of $10 \mathrm{BM}$ samples, whereas culture gave positive results in 8 of 8 cases. PCR-RFLP analysis for Leishmania species identification was performed for all patients and showed a single 250-bp band suggestive of $L$ infantum infection (Fig 2). Eight Leishmania stocks isolated in culture from BMAs were identified by isoenzyme analysis as L infantum zymodeme MON1.

The patients were monitored during and after treatment for a median of 9 months (range: 6-12 months). The PB parasitemia detected by PCR was short-lived, and in all patients the assay became negative after a median of 6 days. However, the clearance of PB parasitemia was more rapid and statistically significant in the patients treated with liposomal amphotericin than in those treated with meglumine antimoniate (median: 6 days vs 14 days; $P<$.03: Mann-Whitney $U$ test). Leishmania PCR on $\mathrm{PB}$ remained negative during the follow-up in all but 1 patient (who is described in greater detail below).

During the follow-up anti-Leishmania serology was unrevealing; in fact, 1 month after the first diagnosis, antibody titers remained unchanged (4 patients) or showed a slight decrease (6 patients). At the last follow-up visit, serology reverted to negativity in 1 patient only, whereas the antibody titers were still unchanged in 2 children, had increased in 2 , and had decreased in the remaining 5 patients.

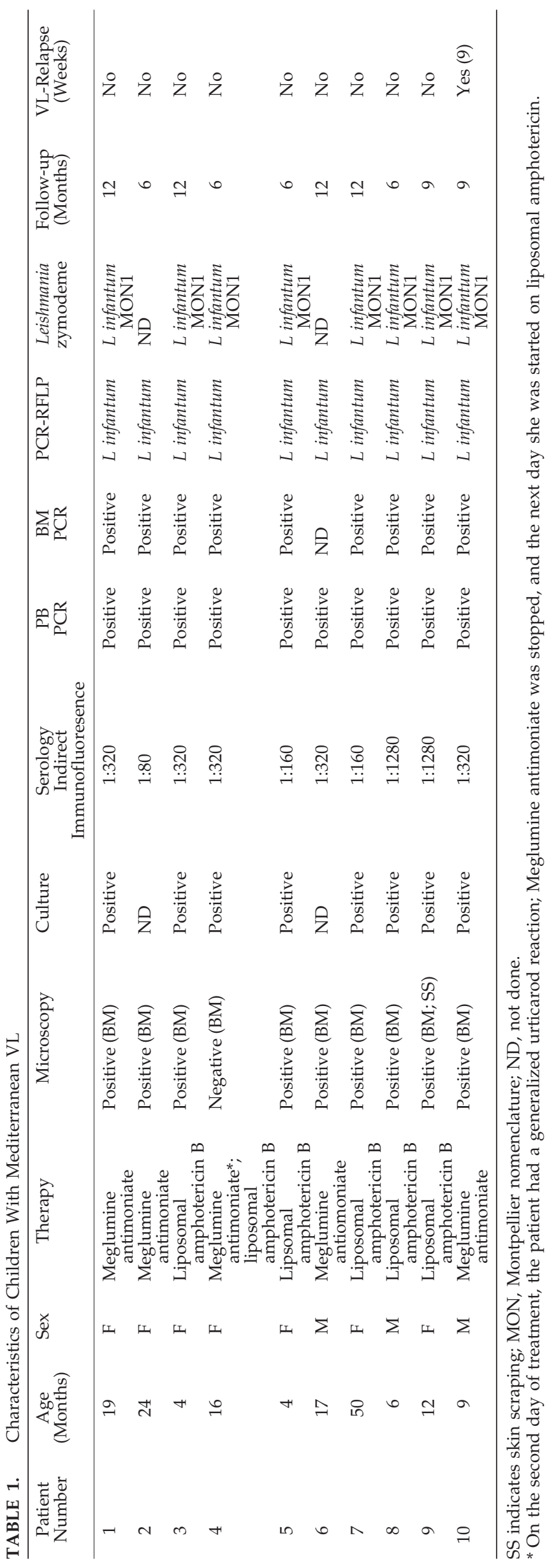




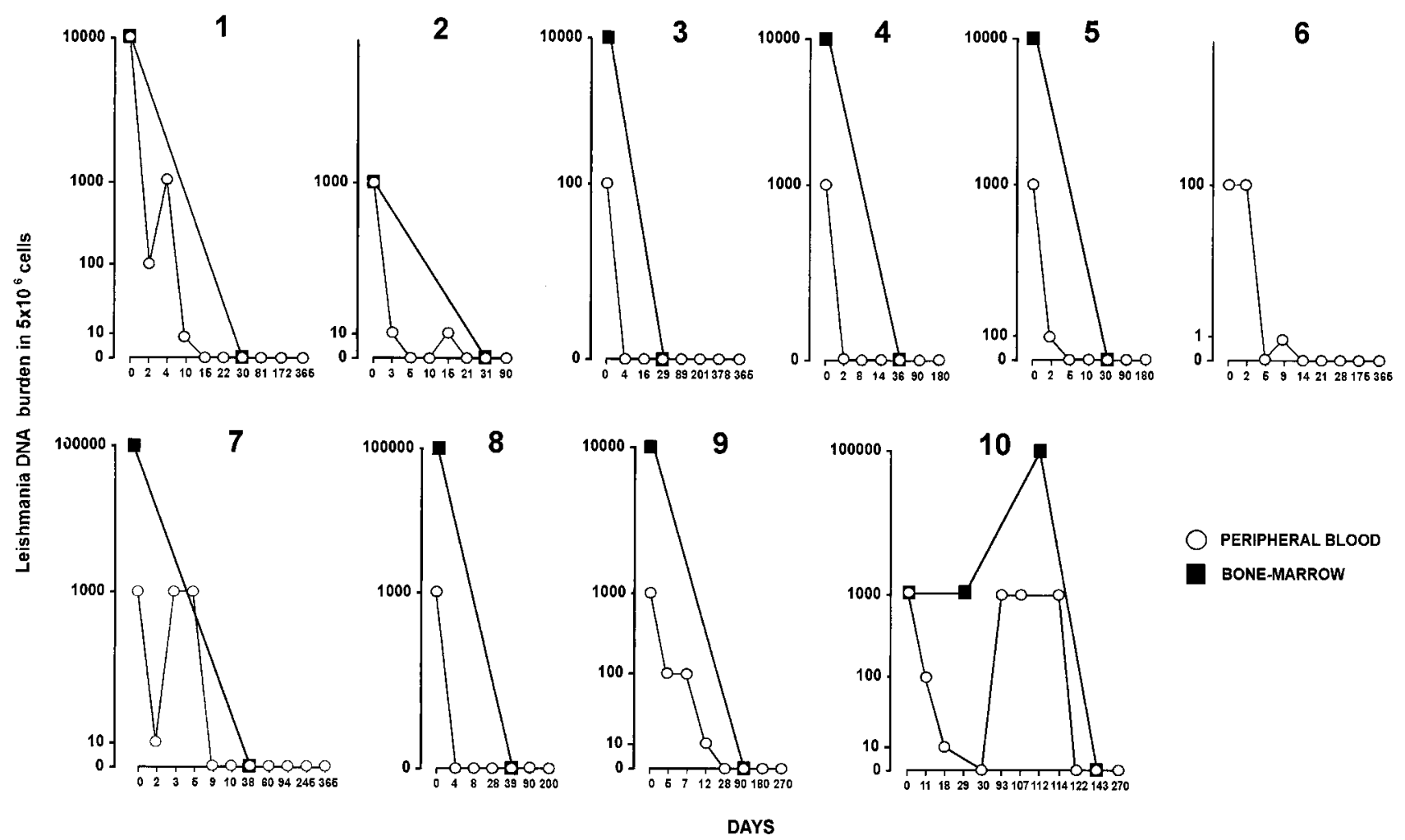

Fig 1. Kinetics of $L$ infantum DNA disappearance and relapse in PB and BM of 10 children with VL under specific therapy as determined by PCR.

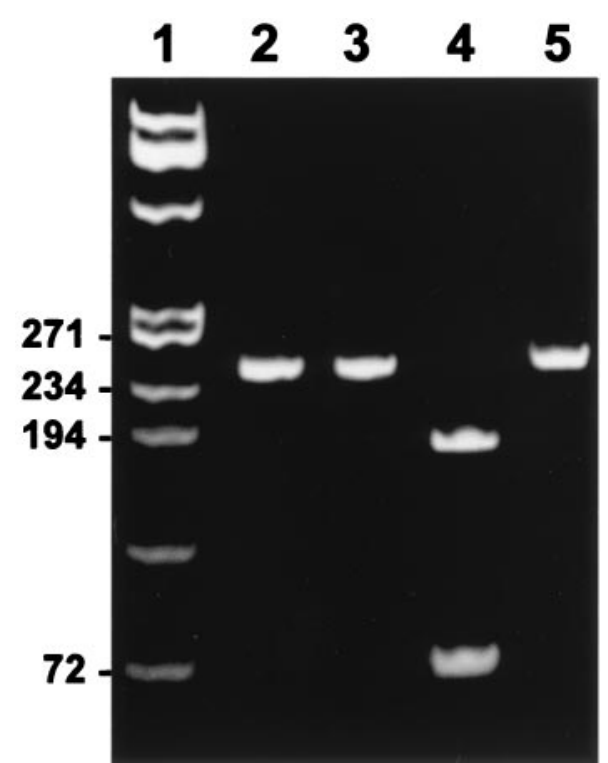

Fig 2. RFLP analysis of a Leishmania-specific nuclear repetitive genomic sequence amplified in vivo from $\mathrm{PB}$ of infected children by PCR. PCR products were purified and digested with the restriction enzyme HaeIII. Lane 1 is a molecular weight marker. Lanes 2 and 3 represent 2 children with $L$ infantum parasitemia. Lanes 4 and 5, respectively, were obtained after digestion of PCR products using DNA extracted from in vitro isolated $L$ donovani and $L$ infantum reference strains.

\section{Case 10}

A 9-month-old child was admitted to our ward with a 15 -day history of high fever $\left(40^{\circ} \mathrm{C}\right)$ and cough. On physical examination, he was febrile and pale, and had an enlarged liver $(5 \mathrm{~cm}$ below the right costal margin), and splenomegaly $(8 \mathrm{~cm}$ below the left costal margin). Laboratory values were as follows: hemoglobin: $8.7 \mathrm{~g} / \mathrm{dL}$; platelets $98.000 / \mu \mathrm{L}$; and C-reactive protein: $2 \mathrm{mg} / \mathrm{dL}$. Leishmania serology was positive at a titer of 1:320; a BMA showed several Leishmania amastigotes. The patient was randomized to receive a 28-day course of meglumine antimoniate (60 mg/kg/d, total dose $504 \mathrm{mg}$ ). Defervescence occurred on the sixth day of therapy. At the end of the treatment PB PCR was negative, the hematologic values had risen $(\mathrm{Hb} 10.2 \mathrm{~g} / \mathrm{dL}$; platelets $252.000 / \mu \mathrm{L})$, and the hepatosplenomegaly was reduced. No culture was performed, but microscopic examination of a posttreatment BMA indicated parasitologic cure and so the patient was discharged despite the fact that PCR on BM was still positive. At the first follow-up visit (third month), the child was in apparent good health and still afebrile, and his weight had increased from $8.4 \mathrm{~kg}$ to $12 \mathrm{~kg}$. At this time, PCR on PB was again positive. Fifteen days later, the child was readmitted to our hospital with persistent fever $\left(39^{\circ} \mathrm{C}\right)$, an enlarged spleen $(5 \mathrm{~cm}$ below the left costal arch), and anemia (Hb $8.8 \mathrm{~g} / \mathrm{dL})$. An additional BMA showed numerous intracellular and extracellular Leishmania amastigotes; the PB and BM PCRs were still positive with the latter showing an increase of parasite load in comparison with the pretreatment sample (Fig 1). The patient was treated with liposomal amphotericin B (Ambisome $3 \mathrm{mg} /$ $\mathrm{kg} / \mathrm{d}$ ) for 10 days and showed defervescence on the third day. PCR on PB became negative 1 week after the start of treatment. One month later, microscopy of BM smears was negative and both BM and PB 
were PCR-negative; at the last follow-up visit (4 months after the new treatment) PB PCR was still negative.

\section{DISCUSSION}

PCR on PB, BM, and lymph node aspirates has been increasingly used as a rapid, sensitive, and specific method for the diagnosis of VL mainly in immunocompromised patients. ${ }^{7-9,13}$ In immunocompetent host, Nuzum et $\mathrm{al}^{14}$ have reported a $90 \%$ sensitivity of PB PCR in parasitologically confirmed cases of kala-azar from 2 areas endemic for Leishmania donovani (India and Africa) and 1 endemic for Leishmania chagasi (Brazil). Other researchers from Sudan detected Leishmania DNA by PCR in the PB of $70 \%$ of their patients with confirmed VL. ${ }^{15}$

To the best of our knowledge, the use of PCR performed on PB (whole blood and buffy coat) and BMA for the diagnosis of VL in pediatric patients has been previously reported in the case of 13 children from France ${ }^{5,8}$ and 12 from China. ${ }^{16}$ In the former study, Minodier et $\mathrm{al}^{5}$ detected Leishmania DNA by PCR in 5 of 5 BM samples and 3 of 7 PB samples taken from 8 children with VL. In a more recent study from France, ${ }^{17}$ the investigators reported concordant and positive PCR results on PB and BM samples from 3 immunocompetent children (in 1 case, the cultures remained negative and diagnosis was confirmed by other methods), and concordant positive PCR and culture results in 1 patient for whom only PB was available. In the study conducted in China, primary or nested PCR was positive on 9 of $10 \mathrm{BM}$ and in 7 of $11 \mathrm{~PB}$ (buffy coat) specimens.

The aims of our study were to compare the results of PB and BM PCR with those of standard parasitologic methods of diagnosing VL and, mainly, to evaluate the usefulness of this molecular method in assessing definitive parasitologic cure after treatment. Our results confirm the reliability of Leishmania PCR performed on $\mathrm{PB}$ in the diagnosis of $\mathrm{VL}$; the high level of sensitivity was reflected by the ability of the assay to detect parasite DNA in PB of patients with VL in all cases, a result that was better than microscopy and equal to culture performed on BMAs.

Our results also show that parasitemia in $\mathrm{PB}$ is usually short-lived in immunocompetent children receiving antileishmanial treatment (a median of 6 days to achieve PCR negativity). In addition, we demonstrated that the persistence of a positive BM PCR is followed by the rapid reappearance of Leishmania parasites in PB and, ultimately, clinically overt disease relapse. As expected, in the majority of cases (all but 2 patients), the PB parasite load was less than in $\mathrm{BM}$; furthermore, the rapid disappearance of $\mathrm{PB}$ parasitemia in this group of immunocompetent children contrasts with what we have previously observed in immunocompromised HIV-positive patients, in whom PB parasitemia became negative under treatment after a median of 5 weeks. ${ }^{9}$ Although in our study the number of treated children with the 2 regimes was limited, we observed a statistically significant more rapid clearance of blood parasitemia in the group assigned to liposomal am- photericin B; however, larger series of patients should be examined before any definitive conclusions can be drawn concerning the relative efficacy of either drug.

It has long been accepted that few or no circulating Leishmania parasites are present in patients with Mediterranean VL. However, a number of recent studies have reported the detection L infantum in PB of patients coinfected with HIV. ${ }^{18-20}$ Our study confirms the frequent presence of circulating parasites during the course of VL also in immunocompetent individuals ${ }^{17}$ and extends this finding to immunocompetent children. Moreover, PCR-RFLP analysis identified the etiologic agent as $L$ infantum in all cases, a finding later confirmed by the standard method of Leishmania biochemical characterization.

Physicians are probably still not confident about replacing $\mathrm{BM}$ examination with PB PCR for the diagnosis of VL. However, we believe that this method should be considered the gold standard for monitoring parasitologic cure because it is easy to perform, noninvasive (thus allowing frequent sampling), well accepted by patients (especially children), and more reliable than microscopy and serology, and more rapid than traditional culture. Finally, patient monitoring by means of Leishmania-specific PCR in the blood can predict treatment failure in a timely manner, and thus allow prompt and appropriate therapeutic adjustments.

\section{ACKNOWLEDGMENTS} $60 \%)$

This work was supported, in part, by a grant from MURST (ex

\section{REFERENCES}

1. World Health Organization. Control of the Leishmaniases. Geneva Switzerland: WHO; 1990. Technical Report Series 793

2. Gradoni L, Bryceson A, Desjeux P. Treatment of Mediterranean visceral leishmaniasis. Bull World Health Organ. 1995;73:191-197

3. Gradoni L, Scalone A, Gramiccia M, Troiani M. Epidemiological surveillance of leishmaniasis in HIV-1-infected individuals in Italy. AIDS. 1996;10:785-791

4. Cascio A, Gradoni L, Scarlata F, et al. Epidemiologic surveillance of visceral leishmaniasis in Sicily, Italy. Am J Trop Med Hyg. 1997;57:75-78

5. Minodier P, Piarroux R, Garnier JM, Unal D, Perrimond H, Dumon H. Pediatric visceral leishmaniasis in southern France. Pediatr Infect Dis J. 1998;17:701-704

6. Maltezou HC, Siafas C, Mavrikou M, et al. Visceral leishmaniasis during childood in southern Greece. Clin Infect Dis. 2000;31:1139-1143

7. Piarroux R, Gambarelli F, Dumon H, et al. Comparison of PCR with direct examination of bone marrow aspiration, myeloculture, and serology for diagnosis of visceral leishmaniasis in immunocompromised patients. J Clin Microbiol. 1994;32:746-749

8. Lachaud L, Dereure J, Chabbert E, et al. Optimized PCR using patient blood samples for diagnosis and follow-up of visceral leishmaniasis, with special reference to AIDS patients. J Clin Microbiol. 2000;38:236-240

9. Pizzuto M, Piazza M, Senese D, et al. Role of PCR in diagnosis and prognosis of visceral leishmaniasis in patients coinfected with human immunodeficiency virus type 1. J Clin Microbiol. 2001;39:357-361

10. Van Eys GJJM, Shoone GJ, Kroon NCM, et al. Sequence analysis of small subunit ribosomal RNA genes and its use for detection and identification of Leishmania parasites. Mol Biochem Parasitol. 1992;51133-142

11. Minodier P, Piarroux R, Gambarelli F, Joblet T, Dumon H. Rapid identification of causative species in patients with Old World leishmaniasis. J Clin Microbiol. 1997;35:2551-2555

12. Rioux JA, Lanotte G, Serres E, Pratlong F, Bastien P, Perieres J. Taxonomy of Leishmania. Use of isoenzymes, suggestion for a new classification. Ann Parasitol Hum Comp. 1990;65:111-125

13. Costa JM, Durand R, Deniau M, et al. PCR enzyme-linked immunosorbent assay for diagnosis of leishmaniasis in human immunodeficiency 
virus-infected patients. J Clin Microbiol. 1996;34:1831-1833

14. Nuzum E, White F III, Thakur C, et al. Diagnosis of symptomatic visceral leishmaniasis by use of the polymerase chain reaction on patient blood. J Infect Dis. 1995;171:751-754

15. Osman OF, Oskam L, Zijlstra EDE, et al. Evaluation of PCR for diagnosis of visceral leishmaniasis. J Clin Microbiol. 1997;35:2454-2457

16. Katamura K, Kawazu SI, Naya T, et al. Diagnosis of kala-azar by nested PCR based on amplification of the Leishmania mini-exon gene. J Clin Microbiol. 1998;36:2173-2177

17. Le Fichoux Y, Quaranta JF, Aufeuvre JP, et al. Occurrence of Leishmania infantum parasitemia in asymptomatic blood donors living in an area of endemicity in southern France. J Clin Microbiol. 1999;37:1953-1957

18. Izri MA, Deniau M, Briere M, et al. Leishmaniasis in AIDS patients: result of leukocytoconcentration, a fast biological method of diagnosis. Bull World Health Organ. 1996;74:91-93

19. Martinez P, De la Vega E, Laguna F, et al. Diagnosis of visceral leishmaniasis in HIV-infected individuals using peripheral blood smears. AIDS. 1993;7:227-230

20. Dereure J, Quaranta JF, Aufeuvre JP, et al. Haemoculture as a tool for diagnosing visceral leishmaniasis in HIV-negative and HIV-positive patients: interest for parasite identification. Bull World Health Organ 1998;76:203-206 